\title{
New Performance Optimization Approach for Cognitive Radio Energy Detection
}

\author{
Patrick Dany Bavoua Kenfack¹, Fabrice Kwefeu Mbakop², Thomas d'Aquin Biyindi ${ }^{3}$ \\ ${ }^{1}$ Department of Electrical and Telecommunications Engineering, National Advanced Polytechnic School, University of Yaounde I, \\ Yaounde, Cameroon \\ ${ }^{2}$ Department of Renewable Energy, National Advanced Polytechnic School, University of Maroua, Maroua, Cameroon \\ ${ }^{3}$ Department of Civil Engineering, Higher Institut of Science, Arts and Crafts, Yaounde, Cameroon \\ Email: danybavoua@gmail.com, mbakop.fabrice@yahoo.fr, biyindithomasdaquin@gmail.com
}

How to cite this paper: Kenfack, P.D.B., Mbakop, F.K. and d'Aquin Biyindi, T. (2020) New Performance Optimization Approach for Cognitive Radio Energy Detection. Open Access Library Journal, 7: e6316.

https://doi.org/10.4236/oalib.1106316

Received: April 11, 2020

Accepted: July 25, 2020

Published: July 28, 2020

Copyright $\odot 2020$ by author(s) and Open Access Library Inc.

This work is licensed under the Creative Commons Attribution International License (CC BY 4.0).

http://creativecommons.org/licenses/by/4.0/

\begin{abstract}
In this paper, we put forward a new method to deal with energy detection low performance in cognitive radio, especially for small values of signal-to-noise ratio. The method is based on a statistical discrimination of received samples in order to improve probability of detection for a given probability of false alarm. We describe how we have determined discrimination criteria with python pandas library, for a signal-to-noise ratio $S N R$ of 0.5 and a number of samples $N s$ of 128 , assuming Gaussian distribution for noise and useful received signals.
\end{abstract}

\section{Subject Areas}

Applications of Communication Systems

\section{Keywords}

Cognitive Radio, Spectrum Sensing, Energy Detection, Probability of Detection $\left(P_{d}\right)$, Probability of False Alarm $\left(P_{f a}\right)$, Receiver Operating Characteristics (ROC), Python Pandas Library

\section{Introduction}

Last few years, we've observed a proliferation of radio standards and connected objects, which lead to a saturation of radio communications spectrum allocation tables. This has resulted in the development of software defined radio and later cognitive radio which is a software radio able to sense its environment and adapt its radio parameters accordingly. The first and key step of cognitive cycle is spectrum sensing. Many spectrum sensing techniques have been developed each with its accuracy and complexity. However, energy detection technique with its 
low complexity remains of great interest despite its low accuracy [1] especially for low signal-to-noise ratio. We propose in this paper a new approach to improve energy detection technique performance while keeping its complexity, this for low signal-to-noise ratio. However, a cognitive radio is a software defined radio able to sense its environment and adapt its radio parameters accordingly. Cognitive radio especially aims to exploit under-utilized spectral resources, by allowing secondary users to temporary use spectrum resources not occupied by primary users [2]. Figure 1 represents the simplified cognitive cycle [3].

The first step of cognitive cycle, spectrum sensing is a key phase of dynamic spectrum access. It allows a secondary user to determine whether a part of spectrum is free or not [4]. The following section is focused on this phase. However, spectrum sensing aims to determine presence of absence of a primary user [5] and thus detect unused spectrum. This spectrum sensing problem can be modeled as a binary hypothesis problem as presented in preceding works such as [3] on energy detection technique for spectrum sensing in cognitive radio [6], on spectrum sensing techniques based on network sensors [7], on enhanced cognitive radio energy detection technique based on estimation of noise uncertainty. Many techniques have been developed for spectrum sensing as for instance: Energy detection, Cyclostationary based detection, Matched Filtering detection, Covariance based detection. However, energy detection remains one of mostly used techniques owing to its low complexity and that it doesn't require any prior information of received signal [8]. The following section presents energy detection technique and its limits.

In Section 2, we present the method. Section 3 is devoted to the performance optimization method proposed for energy detection. In Section 4, the results and discussion are presented. Conclusion appears in Section 5.

\section{Method for Optimizing Energy Detection and Tools}

\subsection{Principle}

Energy detection is done by defining test function $T(y)$ as energy of received sample, and by determining a threshold $\lambda$ such as $T(y)<\lambda$ correspond to $H_{0}$ and $T(y)>\lambda$ correspond to $H_{1}$ [9] [10].

Hence, for $N+1$ received samples $y(k), k=0, \cdots, N$

$$
T(y)=T_{D E}(y)=\sum_{K=0}^{N}|y(k)|^{2}
$$

The performance of such detector is evaluated by following indicators [6].

- Probability of detection $P_{d}$ which represents the probability to decide that a primary user is present when he is really present:

$$
P_{d}=P\left(T(y)>\lambda \mid H_{1}\right)
$$

- Probability of false alarm $P_{f a}$ which represents the probability to decide that a primary user is present whereas the spectrum is free. 


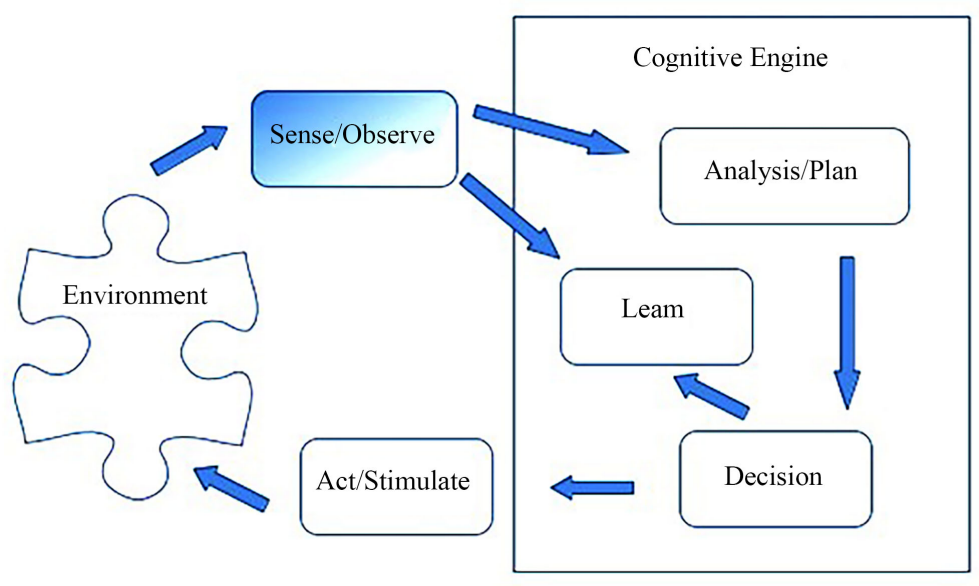

Figure 1. Simplified cognitive cycle [3].

$$
P_{f a}=P\left(T(y)>\lambda \mid H_{0}\right)
$$

Please note: a subsidiary indicator, probability of miss $P_{m}$ can also be used. It represents the probability to decide that the spectrum is free whereas a primary user is present.

$$
P_{m}=1-P_{d}=P\left(T(y)<\lambda \mid H_{1}\right)
$$

In order to compare detectors, $P_{d}$ and $P_{f a}$ can be represented in a single graph called ROC curve (Receiver Operating Characteristic curve), as illustrated in Figure 2.

\subsection{Energy Detection Performance and Limits}

To determine energy detection performance, we consider noise $\omega(k)$ and useful signal $x(k)$ as respectively Gaussian white noise with variance $\sigma_{x}^{2}$ and $\sigma_{x}^{2}$ [6] [12] [13].

Thus $w(k) \sim N\left(0, \sigma_{w}^{2}\right)$ and $x(k) \sim N\left(0, \sigma_{x}^{2}\right)$.

Now, let's consider $N s=N+1$, the number of received samples $y(k), k=0, \cdots, N$. And $S N R=\sigma_{x} / \sigma_{w}$, the signal-to-noise ratio.

\subsubsection{Under $\boldsymbol{H}_{0}$ Hypothesis}

$T_{D E}(y)$ follows a Chi-square distribution with $N s$ degree of freedom.

It can thus be shown by applying central limit theorem that $T_{D E}(y)$ can be approximated by a normal distribution with mean $N s$ and variance $2 N s$.

Thus $T_{D E}(y) \sim N(N s, 2 N s)$ under $H_{0}$ hypothesis.

\subsubsection{Under $\boldsymbol{H}_{1}$ Hypothesis}

$T_{D E}(y)$ follows a non-central chi-square distribution with $N s$ degree of freedom and a non-centrality parameter $N s \times S N R$.

By approximating $T_{D E}(y)$ by normal distribution, it can be shown that under $H_{1}$ hypothesis,

$$
T_{D E}(y) \sim N(N s(S N R+1), 2 N s(2 S N R+1))
$$


ROC plot for Probability of False Alarm vs Probabilty of Detection for different at SNR

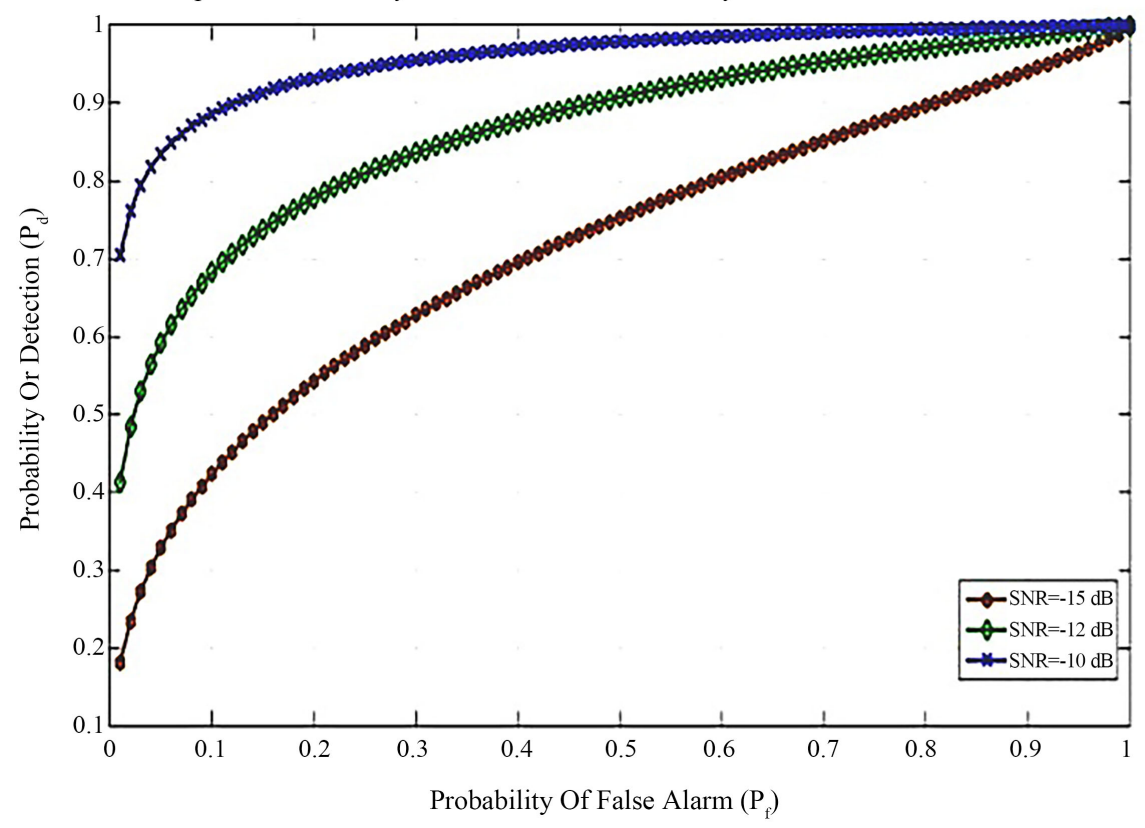

Figure 2. ROC curve illustration [11].

From relations (3) an (4), with can therefore deduce the probability of detection $P_{d}$ and the probability of false alarm $P_{f a}$

$$
\begin{gathered}
P_{d}=P\left(T_{D E}(y)>\lambda \mid H_{1}\right)=1-F_{\chi_{N \mathrm{NS}}^{2}}\left(\frac{2 \lambda}{\sigma_{x}^{2}+\sigma_{w}^{2}}\right) \\
P_{f a}=P\left(T_{D E}(y)>\lambda \mid H_{0}\right)=1-F_{\chi_{\mathrm{NS}}^{2}}\left(\frac{2 \lambda}{\sigma_{w}^{2}}\right)
\end{gathered}
$$

By eliminating $\lambda$ in relations (5) and (6), we obtain the following relation for the ROC curve:

$$
P_{d}=1-F_{\chi_{N s}^{2}}\left(F_{\chi_{N s}^{2}}^{-1} \frac{1-P_{f a}}{1+S N R}\right)
$$

And by approximating (5) and (6) with normal distribution, we have:

$$
\left\{\begin{array}{l}
P_{d}=\frac{1}{\sqrt{2 \pi \times 2 N S(2 N S R+1)}} \int_{\lambda}^{\infty} \exp \left(\frac{-(t-N S(S N R+1))^{2}}{2(2 N S(2 S N R+1))}\right) \\
P_{f a}=\frac{1}{\sqrt{2 \pi \times 2 N S}} \int_{\lambda}^{\infty} \exp \left(\frac{-(t-N S)^{2}}{2(2 N S)}\right)
\end{array}\right.
$$

From relations (10), it appears that $P_{d}$ increases with signal-to-noise ratio $S N R$, for a fiven $P_{f a}$. However, $P_{d}$ quickly decreases for low values of $S N R$. That is a weakness of energy detection despite its low complexity [12] [14]. This technique is less accurate for environment with low signal-to-noise ratio [15]. In the following section, we present our approach to optimize energy detection performance for low $S N R$ by statistical discrimination of received samples. 


\subsection{Performance Optimization with Statistical Discrimination of Samples}

To simplify analysis and present our approach, we will consider for the following a signal-to-noise ratio $S N R=0.5$ and sample number $N s=128$.

Now let's consider noise sample $w(k) \sim N\left(0, \sigma_{w}^{2}\right)$ and useful signal $x(k) \sim N\left(0, \sigma_{x}^{2}\right)$. The received signal is thus $y(k)=x(k)+w(k)$.

To analyze $w$ and $x+w$ characteristics, we've used python pandas library in two steps: first, sample data generation and after true statistical analysis. The choice of python was driven by the fact that, first of all, it's open source, furthermore it can be easily deploy in a software defined radio environment such as GNU Radio.

The following algorithm has been used for sample data generation.

\# Parameters definition

Ns $\leftarrow$ Define Number of sample

\# Please note: If samples are defined from 0 to $\mathrm{N}, \mathrm{Ns}=\mathrm{N}+1$

$\mathrm{M} \leftarrow$ Define the number of $\mathrm{y}$ realization to generate

$\#(\mathrm{y}=\mathrm{x}+\mathrm{w})$

wvar $\leftarrow$ Define the noise variance

$\mathrm{SNR} \leftarrow$ Define signal-to-noise ratio

\# Noise generation

$\mathrm{w} \leftarrow$ Define $\mathrm{w}$ as a Ns $\mathrm{x} M$ matrix

For $\mathrm{k}$ from 0 to $\mathrm{N} \# \mathrm{~N}=\mathrm{Ns}-1$

$\mathrm{w}[\mathrm{k},:] \leftarrow$ Generate $\mathrm{M}$ values following normal distribution $N(0$,wvar $)$

End For

\# Signal generation

$\mathrm{xvar} \leftarrow \mathrm{SNR} *$ wvar \# signal variance computation

$\mathrm{x} \leftarrow$ Define $\mathrm{x}$ as a Ns $\mathrm{x}$ M matrix

For $\mathrm{k}$ from 0 to $\mathrm{N} \# \mathrm{~N}=\mathrm{Ns}-1$

$\mathrm{x}[\mathrm{k}, \mathrm{z}] \leftarrow$ Generate $\mathrm{M}$ values following normal distribution $N(0$,xvar $)$

End For

\# Opening CSV file (Comma Separated Values file) for data recording

f_noise $\leftarrow$ open file 'data_noise.csv' in write mode

f_signal_noise $\leftarrow$ open file 'data_signal_noise.csv' in write mode

\# Header writing in CSV files

Write_csv (f_noise, ['mean', 'std', 'std1', 'std2'])

Write_csv (f_signal_noise, ['mean', 'std', 'std1', 'std2'])

\# Pandas DataFrame generation and data saving

For $\mathrm{j}$ from 0 to $\mathrm{M}-1$

df_noise $\leftarrow$ pandas.DataFrame $(\mathrm{w}[:, \mathrm{j}])$

df_signal_noise $\leftarrow$ pandas.DataFrame $(x[:, j]+w[:, j])$

Write_csv (f_noise, df_noise, df_noise.mean(),

df_noise.std(), df_noise[0:(Ns/2)-1].std(), df_noise[Ns/2 : Ns-1].std())

Write_csv (f_signal_noise, df_signal_noise, 
df_signal_noise.mean(), df_signal_noise.std(),

df_signal_noise[0:(Ns/2)-1].std,

df_signal_noise[Ns/2:Ns-1].std())

End For

\# Closing CSV files

Close file f_noise

Close file f_signal_noise

The previous algorithm allows us to generate and store realizations of noise and received signals (useful signal added to noise) including their mean and variance. Below an extract of samples generated data (Figure 3).

Once we've generated sample data, we can start statistical analysis with python pandas library as detailed below.

\# Loading data from CSV files

import pandas as pd

data_noise $\leftarrow$ pd.read_csv('data_noise.csv')

data_signal_noise $\leftarrow$ pd.read_csv('data_signal_noise.csv')

\# Noise characteristics display

Print 'Noise'

Print data_noise.describe $($ percentiles $=[0.1,0.2,0.8,0.9])$

\# percentiles $=[0.1,0.2,0.8,0.9]$ allow us to determine the distribution at $10 \%$, $20 \%, 80 \%$ and $90 \%$

\# Signal + Noise characteristics display

Print 'Signal + Noise'

Print data_signal_noise.describe $($ percentiles $=[0.1,0.2,0.8,0.9])$

$\#$ percentiles $=[0.1,0.2,0.8,0.9]$ allow us to determine the distribution at $10 \%$, $20 \%, 80 \%$ and $90 \%$

Figure 4 shows the output of this algorithm. We can observe that $80 \%$ of samples of noise have a standard deviation (column std.) below 1.05, whereas less than $20 \%$ of samples of signal + noise have a standard deviation below 1.06. Hence (always assuming $S N R=0.5$ ), a received sample with standard deviation greater than 1.055 (mean of 1.05 and 1.06) has at least a probability of 0.8 to be a $H_{1}$ sample and a maximum probability of 0.2 to be a $H_{0}$ sample.

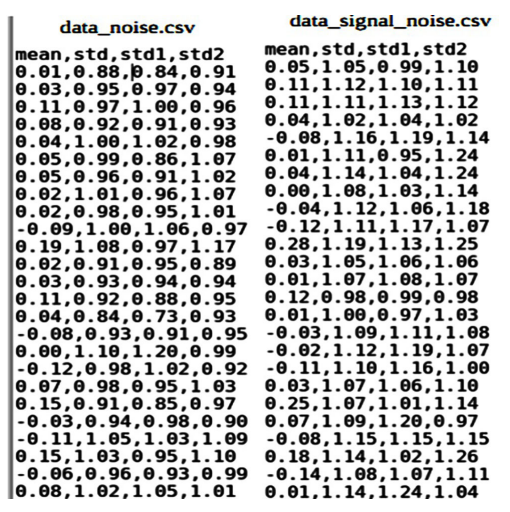

Figure 3. Generation of data samples. 


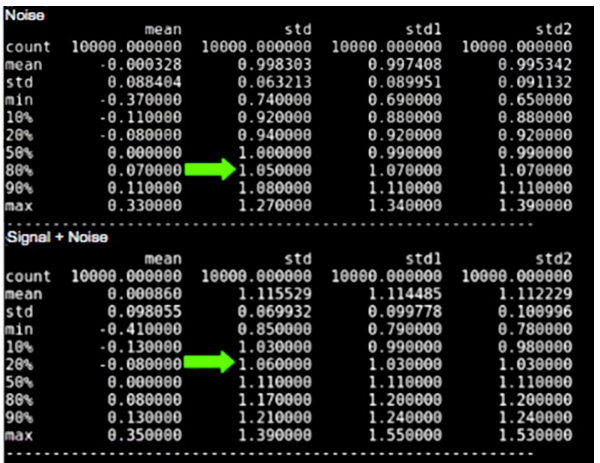

Figure 4. Descriptive statistic of noise and signal + noise samples.

\section{Performance Optimization Method Proposed for Energy Detection}

The diagram in Figure 5 summarizes the performance optimization method proposed for energy detection.

With the previous observation, we thus propose the following algorithm to improve the performance of our energy detection. The idea is to amplify (by applying a factor greater than 1) the computed energy when the received signal has more than $80 \%$ of chance to be $H_{1}$, and to reduce the computed energy (by applying a factor less than 1) when the received signal has more than $80 \%$ of chance to be $H_{0}$.

The proposed Test function (Energy Detection Improved) $T_{E D I}(y)$ is defined by the following rule.

If standard_deviation $(y)>1.055$

ampli $=1.2$

Else If standard_deviation $(y)<1.055$

ampli $=0.8$

Else

ampli $=1$ \# Ambiguous limit case

$$
T_{E D I}(y)=\sum_{k=0}^{N}|\operatorname{ampli} * y(k)|^{2}
$$

where $0<\delta<1$ and $\delta$ is used as a discrimination offset.

\section{Results and Discussion}

\subsection{Algorithm of Simulation}

In this work, Python programming language has been used with its pandas library because it is suitable for scientific and engineering development, for numerical calculations, data analysis and data visualization. However, the proposed test function $T_{E D I}(y)$ can't be easily approximated with central limit theorem like standard energy detection function $T_{E D}(y)$ because of unequal weights applied on samples. Therefore we've used the following algorithm, which is able to draw the ROC curve for any test function $T(y)$. It's based on empirical distribution function. 


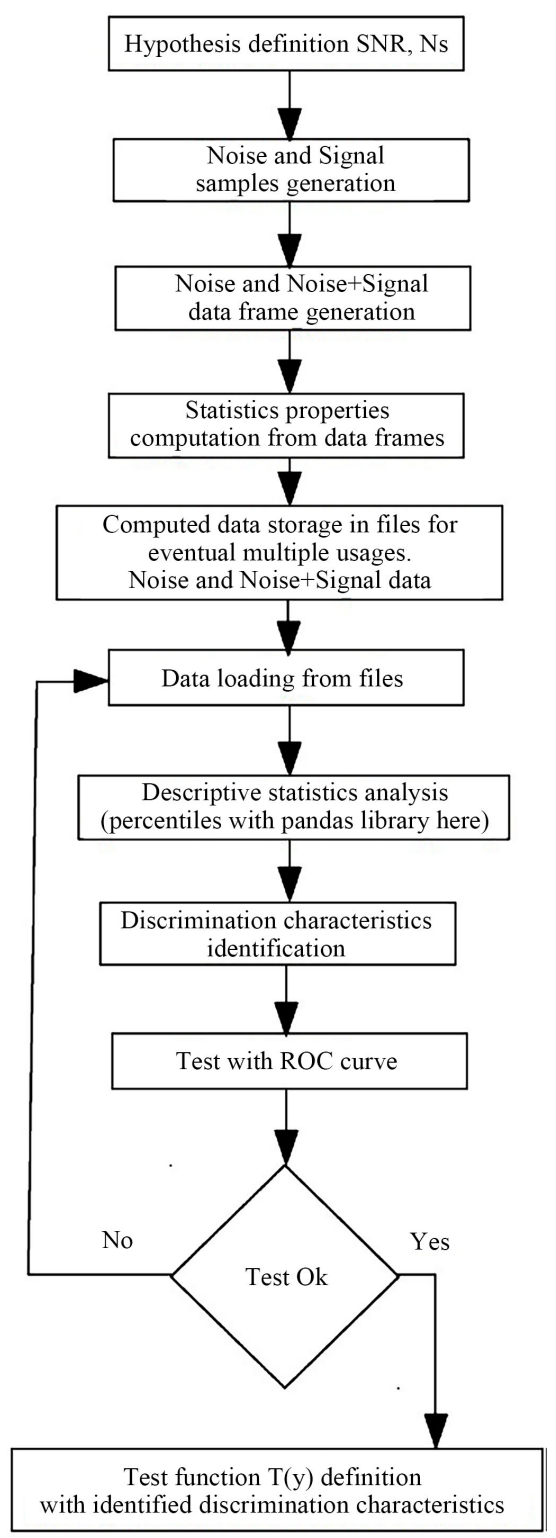

Figure 5. Performance optimization method proposed for energy detection.

\# Parameters definition

$\mathrm{N} s<$ Define the number of samples (to consider for energy computation)

\# Remark: If samples are defined from 0 to $\mathrm{N}, \mathrm{Ns}=\mathrm{N}+1$

$\mathrm{M} \leftarrow$ Define the number of $\mathrm{y}$ realization to generate

wvar $\leftarrow$ Define noise variance

SNR $\leftarrow$ Define signal-to-noise ratio

\# Noise generation

$\mathrm{w} \leftarrow$ Define $\mathrm{b}$ as a Ns $\mathrm{x}$ M matrix

For $\mathrm{k}$ from 0 to $\mathrm{N} \# \mathrm{~N}=\mathrm{Ns}-1$

$\mathrm{w}[\mathrm{k}, \mathrm{:}] \leftarrow$ Generate $\mathrm{M}$ values following normal distribution $N(0$,wvar $)$

End For

\# Signal generationScott, 2014 
$\mathrm{xvar} \leftarrow \mathrm{SNR}$ * wvar \# signal variance computation

$\mathrm{x} \leftarrow$ Define $\mathrm{x}$ as a Ns $\mathrm{x} M$ matrix

For $\mathrm{k}$ from 0 to $\mathrm{N} \# \mathrm{~N}=\mathrm{Ns}-1$

$\mathrm{x}[\mathrm{k}, \mathrm{:}] \leftarrow$ Generate $\mathrm{M}$ values following normal distribution $\mathcal{N}(0$,xvar $)$

End For

\# Generation of $\mathrm{M}$ samples $\mathrm{y}[\mathrm{i}]$ of $\mathrm{y}$

$\mathrm{y} \leftarrow$ Define $\mathrm{y}$ as $\mathrm{M}$ dimension vector

\# Computation under $\mathrm{H}_{0}$ hypothesis

For I from 0 to $\mathrm{M}-1$

$\mathrm{y}[\mathrm{i}] \leftarrow \mathrm{T}(\mathrm{w}[:, \mathrm{i}])$

\# y[i] based on generated noise samples

\# Please note: $\mathrm{w}[$ :, i] samples are taken in column (independent variables)

End For

\# Distribution function under $\mathrm{H}_{0}$ hypothesis

py $0<$ Define py0, probability vector of $M$ dimension

For i from 1 to $\mathrm{M}$

py0 $[\mathrm{i}] \leftarrow \mathrm{i} / \mathrm{M}$

End For

sy0 $\leftarrow$ Sort y value and set result to sy0 variable

\# sy0 and py0 thus represent empirical distribution unction under $\mathrm{H}_{0}$ hypothesis

dfy $0 \leftarrow$ interpolation function $($ sy 0 , py 0$)$ with minimal value 0 and maximal value 1

\# Computation under $\mathrm{H}_{1}$ hypothesis

For i from 0 to $M-1$

$\mathrm{y}[\mathrm{i}] \leftarrow \mathrm{T}(\mathrm{x}[:, \mathrm{i}]+\mathrm{w}[:, \mathrm{i}])$

\# y[i] based on generated signal and noise samples

\# Please note: $\mathrm{x}[:, \mathrm{i}]$ and $\mathrm{w}[:, \mathrm{i}]$ samples are taken in column (independent variables)

End For

\# Distribution function under $\mathrm{H}_{1}$ hypothesis

py $1 \leftarrow$ Define py 1 , probability vector of $M$ dimension

For i from 1 to $\mathrm{M}$

pyl $[\mathrm{i}] \leftarrow \mathrm{i} / \mathrm{M}$

End For

sy $1 \leftarrow$ Sort y value and set result to sy1 variable

\# syl and pyl thus represent empirical distribution function under $\mathrm{H}_{1}$ hypothesis

dfy $1 \leftarrow$ interpolation function $($ sy 1 , py 1$)$ with minimal value 0 and maximal value 1

\# $\mathrm{P}_{\mathrm{fa}}$ and $\mathrm{P}_{\mathrm{d}}$ determination

lambda $<$ array from 0 to $10000^{\star} \mathrm{Ns}$ with step of $100^{\star} \mathrm{Ns}$

Pfa $=1-$ dfy0(lambda)

Pd = 1 - dfy1(lambda)

\# ROC Curve drawing

Plot(Pfa, Pd, label = 'ROC Curve') 


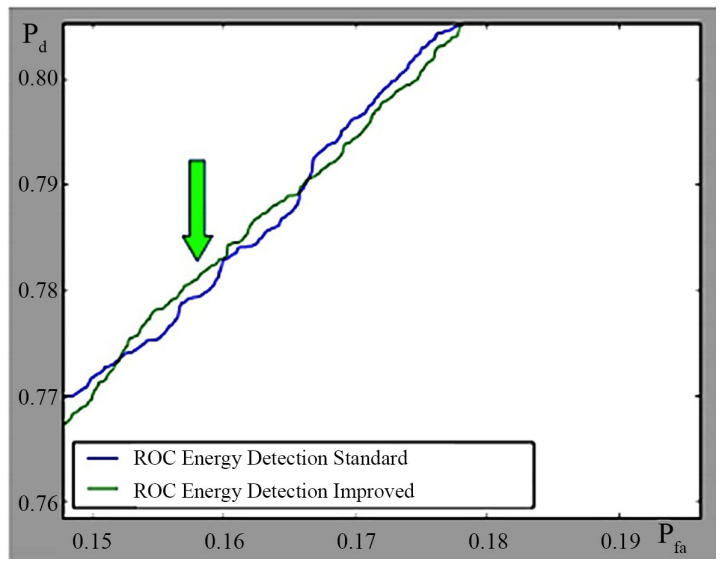

Figure 6. ROC standard and improved energy detection.

\subsection{Comparison of ROC Energy Detection Standard and ROC Energy Detection Improved}

Figure 6 presents the result after the simulation of the improved algorithm.

The simulation of the ROC curve of this energy detection method with statistical optimization shows that we can obtain for some values of probability of false alarm $P_{f a}$ a probability of detection $P_{d}$ greater than standard energy detection one. Figure 5 illustrates an example with $\delta=0.2$. We can observe from simulation that improved energy detection ROC curve is not all the time above the standard one. But, asshown by the green arrow, the result is interesting when wecan have the improved ROC curve, upper for low values of $P_{f a}$ since in real world; the objective is to minimize probability of false alarm $P_{f a}$ while increasing probability of detection $P_{d}$.

\section{Conclusion}

Energy detection remains one of the mostly used spectrum sensing technique despite its low performance, especially for low signal-to-noise ratio. We propose in this paper a new approach to improve energy detection performance for low signal-to-noise ratio, and we've simulated the proposed algorithm for a signal-to-noise ratio of 0.5 . We've seen on the ROC curve that it's possible to have a probability of detection $P_{d}$ greater than the one of standard energy detection, for some low values of probability of false alarm $P_{f a}$. Future works may be interested to generalize the method for any value of signal-to-noise ratio.

\section{Conflicts of Interest}

The authors declare no conflicts of interest regarding the publication of this paper.

\section{References}

[1] Meghana, V. and Vemula, S. (2015) Energy Detection Sensing of Unknown Signals over Fading Channels. International Journal of Scientific Engineering and Technology Research, 4, 5727-5731. 
[2] Axell, E., Geert, L., Erik, G. and Poor, H.V. (2012) State-of-the-Art and Recent Advances Spectrum Sensing for Cognitive Radio. IEEE Signal Processing Magazine, 29, 101-116. https://doi.org/10.1109/MSP.2012.2183771

[3] Abdulsattar, M.A. and Zahir, A.H. (2012) Energy Detection Technique for Spectrum Sensing in Cognitive Radio: A Survey. International Journal of Computer Networks and Communications, 4, 223-242. https://doi.org/10.5121/ijcnc.2012.4514

[4] Harpreet, K. and Jyoti, S. (2015) A Comparison of Improvements in Spectrum Sensing Methods in CognitiveRadio Using Various Techniques. International Journal of Engineering Research and General Science, 3, 164-169.

[5] Samriti, K. (2015) Spectrum Sensing Techniques and Challenging Issues in Cognitive Radio. SSRG International Journal of Electronics and Communication Engineering, 2, 25-29.

[6] Men, S.Y. (2016) Spectrum Sensing Techniques in Cognitive Wireless Sensor Networks. PhD Dissertation, University of Nantes, Nantes.

[7] Deepika, J., Amanpreet, K. and Swaran, A. (2016) Enhanced Cognitive Radio Energy Detection Technique Based on Estimation of Noise Uncertainty. International Journal of Advanced Research in Computer Engineering \& Technology, 5, 1339-1342.

[8] Deepa, N.R., Ravinder, Y. and Chetna, S. (2016) Testbed Implementation of MultiDimensional Spectrum Sensing Schemes for Cognitive Radio. ICTACT Journal on Communication Technology, 7, 1289-1294.

[9] Herath, S.P., Rajatheva, N. and Tellambura, C. (2011) Energy Dectection of Unknown Signals in Fading and Diversity Reception. IEEE Transactions on Communications, 59, 2443-2453. https://doi.org/10.1109/TCOMM.2011.071111.090349

[10] Scott, P. (2014) Literature Review of Cognitive Radio Spectrum Sensing. EE 359 Project, Stanford University, Stanford.

[11] Pranav, P. and Arpita, P. (2015) The Harvest of Energy Detection Adjunct Spectrum Sensing Is Analyzed Using ROC Curves. International Journal of Engineering Research \& Technology, 4, 334-339. https://doi.org/10.17577/IJERTV4IS050358

[12] Saman, A., Chintha, T. and Hai, J. (2014) Energy Dectection for Spectrum Sensing in Cognitive Radio. Springer, New York.

[13] Jasleen, K. and Khan, S.A. (2015) Investigate the Performance of Spectrum Sensing Cognitive Radio Users through Energy Detection. International Journal of Emerging Research in Management \& Technology, 4, 203-209

[14] Maninder, S., Pradeep, K., Anusheetal and Sandeep, K.P. (2016) Techniques for Spectrum Sensing in Cognitive Radio Networks: Issues and Challenges. International Research Journal of Engineering and Technology, 3, 198-205.

[15] Roopali, G. and Nitin, S. (2016) Current Trends and Research Challenges in Spectrum-Sensing for Cognitive Radios. International Journal of Advanced Computer Science and Applications, 7, 402-408. https://doi.org/10.14569/IJACSA.2016.070756 